\title{
Fairness in Multi-Operator Energy Sharing
}

\author{
Maria Oikonomakou ${ }^{\dagger}$, Angelos Antonopoulos ${ }^{\diamond}$, Luis Alonso ${ }^{\star}$, Christos Verikoukis ${ }^{\diamond}$ \\ $\dagger$ Iquadrat Informatica, Barcelona, Spain \\ ${ }^{\diamond}$ Telecommunications Technological Centre of Catalonia (CTTC/CERCA), Castelldefels, Spain \\ ${ }^{\star}$ Technical University of Catalonia (UPC-BarcelonaTECH), Signal Theory and Communications Department, \\ Castelldefels, Spain \\ Email: moikonomakou@iquadrat.com, \{aantonopoulos, cveri\}@cttc.es, luisg@tsc.upc.edu
}

\begin{abstract}
The present paper studies fair sharing possibilities of stored renewable energy (RE) in densely populated areas among multiple mobile network operators (MNOs). The main objective is to enable MNOs, who already share their infrastructure in terms of a green network operation, to further reduce their power consumption by fairly sharing (i) the solar power harvested during daylight and (ii) the harvested energy that is stored in storage devices, e.g. batteries, during night hours. To this end, a bankruptcy game is designed so as to capture the energy sharing interactions among MNOs. Taking into consideration the energy deficits of each MNO for a studied period of time, Shapley Value (SV) is employed to fairly determine the amounts of energy to be allocated to each MNO. Focusing on night hours, it is shown that the proposed scheme provides all cooperative MNOs with long store-energy-based network operation during night in comparison to baseline schemes. Moreover, we display that the proposed scheme outperforms its counterparts in terms of needed grid support and allocation fairness among the cooperative MNOs for different MNO traffic patterns and volumes.
\end{abstract}

Index Terms-Energy sharing, network sharing, renewable energy, bankruptcy theory, Shapley Value.

\section{INTRODUCTION}

An enormous increase in traffic demands is expected by 2020, according to recent studies [1]. Mobile network operators (MNOs) densify their infrastructure in order to support their demands, leading though to increased energy consumption and carbon dioxide $\left(\mathrm{CO}_{2}\right)$ emissions. With information and communication technology infrastructure (ICT), especially base stations, held responsible for severely contributing to the problem [2], many works on energy saving and $\mathrm{CO}_{2}$ emission reductive techniques have been provided in the past [3], [4].

With regard to achieving a greener network operation, works on adoption of renewable energy (RE) sources have also been presented [5], [6]. Introduction of RE sources in the operation of telecommunication networks, not only contributes to the reduction of energy consumption and the carbon footprint, but also provides reliable network coverage in cases of network operation failures, as well as reduced operational expenses. Aiming at minimizing usage of grid electricity, industry has also embraced RE sources by proceeding to the deployment of zero-energy sites, such as Ericsson ${ }^{1}$ and Nokia ${ }^{2}$.

RE sources have a generation profile that is difficult to predict due to its dependency on weather conditions and choice of geographical location. Solar energy minimizes to

\footnotetext{
${ }^{1}$ https://www.ericsson.com/ourportfolio/products/zero-site

${ }^{2}$ https://networks.nokia.com/products/zero-emission
}

a certain degree the uncertainty of energy generation, thanks to daylight periodicity. Moreover, storage devices, such as batteries and grid connection are usually used to counteract $\mathrm{RE}$ generation randomness. However, both aforementioned solutions come at a price. On the one hand, grid connection inserts additional expenses for energy purchasing. On the other hand, even though storage devices provide free energy, their deliverable amount of energy is strictly predefined. In a single operator or rural environment, where traffic load demands are of lower volume, limits of storage capability would not be an issue. However, the expected increase in the traffic load, along with the, dominating in dense urban areas, multioperator environments, have brought forward energy cost and limited storage as issues that seek for a solution.

Towards this direction, energy sharing techniques have been presented by the research community. Energy sharing among elements of the same network has been studied in the past extensively in various forms: via power lines [7], via grid based on technical characteristics [8] or via aggregator-assisted energy transfer [9], [10]. Energy trading with the grid has also been presented as an easy energy and cost management solution, using criteria such as RE technical characteristics [11] or with an aggregator being used as an impartial entity [6]. Although state-of-art research has covered energy sharing and trading among network elements or with the grid in single operator environments, fewer are the works referring to multioperator ones. Recently, [12] presented energy transactions in a multi-MNO environment. However, no RE generation or storage device is assumed and considered MNOs deal only with the hybrid energy market.

In this paper, we focus on sharing techniques of energy among multiple MNOs with collocated shared network infrastructure and encourage cooperation among them by studying relative fairness issues. More specifically, in order to avoid extra expenses imposed by trading with the grid, we assume RE infrastructure of solar energy, accompanied by a storage device that is connected via power lines with all MNO networks. In this context, MNOs share the harvested energy to cover their energy needs throughout the day and have to share the strictly determined amount of stored energy for the night hours. Thus, an allocation problem of gathered or stored RE by cooperative MNOs arises so as to not only reduce grid-energy dependency, but also to achieve an overall fair energy management. To this end, we formulate and solve our problem as a bankruptcy game, which is a tool that certifies 


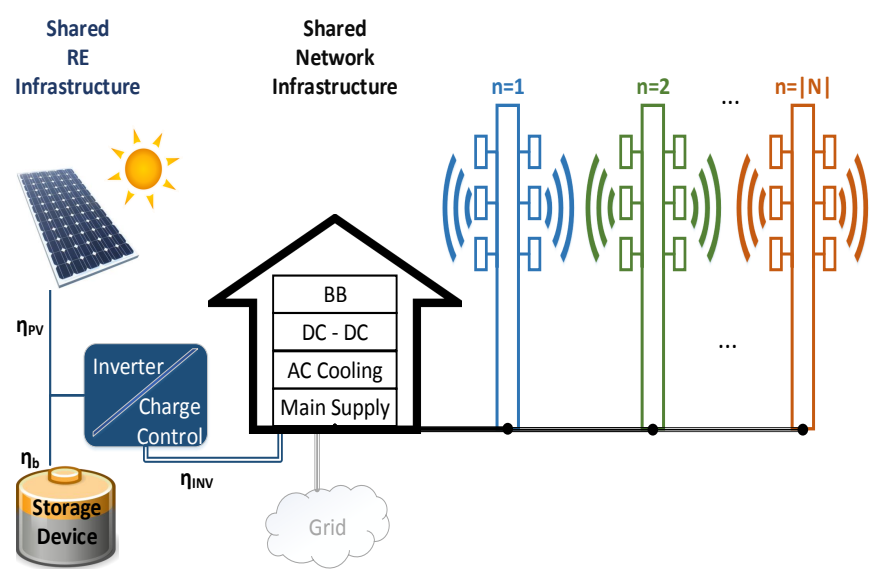

Figure 1. System model.

the complete allocation of a certain amount of entity among a predefined number of cooperative agents. Moreover, we use Shapley Value to reassure fairness for the allocation problem. We study the period of network operation based on harvested or stored RE for the proposed scheme in contrast to baseline methods, the percentage of grid-energy bought in each case, as well as raised fairness issues related to each allocation strategy.

The rest of our paper is organised as follows: Section II describes the adopted system model. In Section III, we explicitly describe our problem, formulate it and present our proposed solution. The simulation scenario along with the respective results are presented in Section IV. Finally, Section $\mathrm{V}$ concludes this paper.

\section{SySTEM MODEL}

In the present section, we describe the system model and technical details considered for the network configuration.

\section{A. System Model and Operation}

We focus our study on a densely populated macrocellsized area, $A$, where $\mathcal{N}$ MNOs have located their Long Term Evolution Advanced (LTE-A) infrastructure, as depicted in Fig. 1. The network of each MNO $n \in \mathcal{N}$ is composed of one eNodeB (eNB) located in the centre of $A$. For the sake of facilitated notation, we will use index $n \in \mathcal{N}$ interchangeably for MNOs and respective eNBs. We assume passive network infrastructure sharing among $\mathcal{N}$, i.e., sharing of the network power consumption that is attributed to cooling and signal processing procedures. Assuming that $A$ is a densely populated area with a traffic pattern $f(t)$ as indicated in [13], we assume the respective traffic profiles, $f_{n}(t)=a_{n} f(t), a_{n} \in \mathbb{R}_{++}$for each $n \in \mathcal{N}$, with uniform user equipment (UE) arrival in $t$ and uniform distribution in $A$. Each UE $k$ has a specific guaranteed bit rate demand, $r_{k}$ and can be associated to the eNB of its provider MNO $n$, provided that the signalto-interference-plus-noise ratio (SINR) of $k$ with eNB $n$ at $t$, $S I N R_{k n}(t)$, is higher than a certain SINR threshold, $S I N R_{\text {thresh }}$. We define $S I N R_{k n}(t)$ as



where $P_{n}(t)$ is the power consumption of $n$ at $t, g_{k n}$ indicates the channel gain between $k$ and $n$, while $\sigma^{2}$ stands for the noise power level [14]. It is noted that for the calculation of channel gain $g_{k n}$, pathloss and shadowing fading are taken into consideration. Based on [13], we model the power consumption $P_{n}(t)$ of a shared eNB $n$ as

$$
P_{n}(t)=P_{n}^{\text {pass }}+\frac{w_{n}(t)}{W_{n}} G_{T R X} \Delta_{P} P_{n}^{t r},
$$

where $w_{n}$ is the amount of bandwidth of $n$ that is occupied so as to cover the traffic of $n$ in $t$ and $W_{n}$ is the total amount of bandwidth that is allocated to $n$ by default. Also, $G_{T R X}$ is the number of transceiver chains on eNB $n, \Delta_{P}$ is the slope of load-dependent power consumption and $P_{n}^{t r}$ corresponds to the maximum transmit power of $n$. It is noted that passive sharing allows $\mathcal{N}$ to share the constant part of their power consumption, $P_{n}^{\text {pass }}$, as follows

$$
P_{n}^{\text {pass }}=\frac{G_{T R X} P_{n}^{\text {con }}}{|\mathcal{N}|},
$$

where $P_{n}^{c o n}$ represents the power consumption at the minimum non-zero output power [13].

Occupied bandwidth of $n, w_{n}(t)$, is determined by the traffic that $n$ serves at $t$. We focus on the downlink (DL) transmission activity, where orthogonal frequency division multiple access (OFDMA) is employed, with transmission of information in resource block (RB) pairs of $1 \mathrm{~ms}$. Thus, it is

$$
w_{n}(t)=\sum_{k \text { in } \mathcal{K}} q_{k n} \cdot\left\lceil\frac{r_{k}}{W_{n}^{R B} f\left(S I N R_{k n}\right)}\right\rceil,
$$

where $W_{n}^{R B}$ is the bandwidth that corresponds to an RB pair of $n$ and $f\left(S I N R_{k n}\right)$ is the spectral efficiency of the link between $k$ and $n$ [15]. Given the fact that adaptive modulation and coding (AMC) is adopted over any radio link, we map the requested data rate $r_{k}$ and $S I N R_{k l}$ to a respective spectral efficiency as indicated in [14, Table A.2]. We denote with $q_{k n}$ the association state of $k$ with $n$, with $q_{k n}=1$ when $k$ is associated to $n$ and $q_{k n}=0$ otherwise.

\section{B. Solar energy generation}

As can be seen in Fig. 1, we assume that there is RE generation infrastructure in area $A$. We use solar energy as the chosen RE source and model the solar RE generation $h(t)$ of a photovoltaic (PV) panel at $t$ with a sinusoidal profile ${ }^{3}$

$$
h(t)=L \cdot E_{A} \cdot\left(1-\eta_{P V}\right) \cdot \sin (\omega t),
$$

where $L$ is the assumed number of PV panels, $E_{A}$ stands for the average solar generation of a PV panel in area $A$ (Wh/m $/ m^{2} /$ hour $), \eta_{P V} \in[0,1]$ stands for the percentage of PV panel RE losses due to its temperature, cleanness and shading, mismatching operation of elements, wiring and ageing [16]. Lastly, we consider $\omega=\frac{2 \pi}{T_{R E}}$, where $T_{R E}$ is the period of (5).

We assume that the generated solar RE, $h(t)$ can be either be procured from the MNO network system for immediate consumption or stored in a storage device. Procured RE $h(t)$ that reaches the MNO shared network is calculated as

$$
H(t)=h(t) \cdot\left(1-\eta_{I N V}\right),
$$

${ }^{3}$ http://www.elia.be/en/grid-data/power-generation/Solar-power-generationdata/Graph 
where $\eta_{I N V} \in[0,1]$ is the inversion energy losses factor [16].

The RE system is supported with a storage device, which can be composed of one or multiple batteries, depending on the storage necessities of the system. If $M$ is the total number of batteries of nominal voltage $V$ and capacity $I$ composing the storage device, the maximum power that it can store is

$$
B_{\max }=M \cdot V \cdot I \cdot(1-D O D),
$$

where $D O D$ is the maximum level of battery discharge that prevents degradation of battery's health [17].

It is noted that battery inefficiency issues result in a loss of $h(t)$ that is stored in the storage device or that is received by the MNO network shared system. In more detail, the amount of solar energy $b(t)$ that is stored in $t$ is

$$
b(t)=h(t) \cdot\left(1-\eta_{b}\right), b(t) \leq b_{\max },
$$

where $\eta_{b} \in[0,1]$ is the energy losses factor due to battery deficiency [17]. Considering a studied period $T=\left[t_{1}, t_{2}\right]$ and no previous energy procurements, the amount of stored energy $Y(t)$ that the MNO network shared system can receive is

$$
Y(t)=\left(1-\eta_{I N V}\right)\left(b(t<T)+\sum_{t \in T} b(t)\right) .
$$

\section{PROBLEM DESCRIPTION AND PROPOSED SOLUTION}

In the present section we describe our problem in Section III-A, formulate it in Section III-B, and finally present our proposed solution in Section III-C.

\section{A. System Operation}

Provided that each MNO $n$ seeks energy neutrality for the operation of its own network, i.e., its eNB $n$, during a period $T$, its total energy demands during $T$ are

$$
d_{n}(t)=\sum_{t \in T} P_{n}(t) \cdot t
$$

Depending on $(i)$ the energy demands $d_{n}(t)$ of each $n \in \mathcal{N}$ at $t$, (ii) the available amount of usable harvested RE during $T$ and (iii) the usable stored RE at $t$, total MNO energy deficits are covered by different sources. We specify three cases:

- Energy demands are covered by generated RE during $\mathbf{T}\left(\sum_{n \in \mathcal{N}} d_{n}(t) \leq \sum_{t \in T} H(t)\right)$ : Primarily, an MNO $n$ seeks to cover its energy deficits, $x_{n}^{R E}$, using the RE that is generated from the shared solar system during $T$, $\sum_{t \in T} H(t)$. Thus, $x_{n}^{R E}$ is defined as

$$
x_{n}^{R E}=d_{n}(t) .
$$

- Energy demands are covered by generated RE during $\mathbf{T}$ and usable stored energy $\left(\sum_{t \in T} H(t)<\sum_{n \in \mathcal{N}} d_{n}(t) \leq Y(t)+\sum_{t \in T} H(t)\right):$ If $\sum_{t \in T} H(t)$ is insufficient to cover the energy demands of all MNOs, $\sum_{n \in \mathcal{N}} d_{n}(t)$, then MNOs are obliged to share $\sum_{t \in T} H(t)$ among them, receiving certain shares of $\mathrm{RE}, \Phi_{n}^{R E}$ and procure their remaining individual energy deficits, $x_{n}^{\text {store }}$, from the stored energy they can receive at $t, Y(t)$. Thus, $x_{n}^{\text {store }}$ is defined as

$$
x_{n}^{\text {store }}=d_{n}(t)-\Phi_{n}^{R E} .
$$

- Energy demands are covered by generated RE during $\mathbf{T}$, usable stored energy and the grid $\left(Y(t)+\sum_{t \in T} H(t)<\sum_{n \in \mathcal{N}} d_{n}(t)\right):$ In the case that the sum of $\sum_{t \in T} H(t)$ and $Y(t)$ is insufficient to provide zero-grid energy network operation, MNOs are obliged to share both $\sum_{t \in T} H(t)$ and $Y(t)$ among them, receiving certain shares $\Phi_{n}^{R E}$ and $\Phi_{n}^{\text {store }}$, respectively, and buy their remaining individual energy deficits, $x_{n}^{\text {grid }}$, from the grid. Thus, $x_{n}^{\text {grid }}$ is defined as

$$
x_{n}^{\text {grid }}=d_{n}(t)-\Phi_{n}^{R E}-\Phi_{n}^{\text {store }} .
$$

It is noted that $P_{n}(t), H(t)$ and $Y(t)$ are given by eq. (2), (6) and (9), respectively. Moreover, if $T$ coincides with non-solar hours, generation of solar RE is equal to zero. Then, MNOs seek energy neutrality for their eNBs receiving energy from the storage device and when the latter is not enough, demand their energy deficits from the grid. Thus, MNO energy deficits are described by eq. (12) and eq. (13), respectively, with $\Phi_{n}^{R E}=$ 0 . However, as far as $\Phi_{n}^{R E}$ and $\Phi_{n}^{\text {store }}$ are concerned, they need to be more accurately defined by a fair allocation method to preserve MNO cooperation. To this end, we proceed to a detailed problem definition in the next section.

\section{B. Problem Formulation}

System operation of Section III-A introduces a certain issue: a defined entity, i.e., the insufficient solar RE, $\sum_{t \in T} H(t)$ or the insufficient received stored energy, $Y(t)$, have to be totally allocated among a set of MNOs, $\mathcal{N}$, to determine their rightful shares of energy $\Phi_{n}^{R E}$ and $\Phi_{n}^{\text {store }}$, respectively and eventually satisfy their demands, $d_{n}(t)$. It is possible that cooperative MNOs could proceed to an equal allocation of the studied entity among them or proceed to an allocation prioritizing their energy demands. However, such strategies could result into a distribution of energy that would not only be energy inefficient, but also unfair for other MNOs. In our paper, we propose a more cooperative approach that could set aside possible inefficiency and fairness issues and provide an applicable solution to the described problem. To this end, we model the energy allocation problem as a bankruptcy game.

A bankruptcy game is defined by a specific entity that needs to be completely allocated among a group of players [18], [19]. Each of the players makes a claim on the obtained entity, while the sum of the players' claims cannot exceed it. A utility function is set for the game, according to cooperative game theory, which eventually allocates to each of the players part of the entity, i.e., the payoff.

In correspondence to the present case, $\sum_{t \in T} H(t)$ or $Y(t)$ constitute an entity $E$ that has to be fairly and completely allocated among cooperative MNOs. Let $\mathcal{S} \subseteq \mathcal{N}$ be a coalition of MNOs who cooperate in order to receive an amount of harvested or stored RE of the considered $E$, i.e., $\sum_{t \in T} H(t)$ or $Y(t)$, respectively, before buying energy from the grid. Thus, considered entity $E$ needs to be completely allocated to all participants of coalition $\mathcal{S}$, who can be portrayed as the players of the game. Each player $s \in \mathcal{S}$ demands an amount $d_{s}(t)$ of $E$, which constitutes its claim, in accordance to eq. (10). Consequently, we have a bankruptcy problem, $B$, modelled as

$$
B=\left\{\left(E, d_{s} \in \mathbb{R}_{++} \times \mathbb{R}_{+}^{|\mathcal{S}|}\right): E \leq \sum_{s=1}^{|\mathcal{S}|} d_{s}\right\} .
$$


We use coalitional game theory, and more specifically, coalitional games of transferable utility (TU), in order to define the characteristic function, $V_{B}, V_{B}: 2^{N} \times \mathbb{R}$, which evaluates each bankruptcy problem $B$ and associates it to a real value:

$$
V_{B}(\mathcal{S})=\left\{\begin{array}{l}
\min \left\{d_{s \in \mathcal{S}}, E-\sum_{s \notin \mathcal{S}} d_{s}\right\}, \mathcal{S}=\{s\} \\
\min \left\{\sum_{s \in \mathcal{S}} V_{B}(s), E-\sum_{s \notin \mathcal{S}} d_{s}\right\}, \mathcal{S} \neq\{s\} .
\end{array}\right.
$$

It is noted that in the case that $V_{B}(\mathcal{S})<E-\sum_{s \notin \mathcal{S}} d_{s}$, then $V_{B}(\mathcal{S})=0$, since the entity cannot be totally allocated to the cooperative MNOs [18], [19].

Let $\phi_{s}\left(V_{B}(\mathcal{S})\right)$ be the payoff in the case of a $B$ game with utility function $V_{B}$. The payoff $\phi_{s}\left(V_{B}(\mathcal{S})\right)$ represents the amount of harvested or stored RE allocated to a player $s \in \mathcal{S}$, depending on the considered $E$ (described before as $\Phi_{n}^{R E}$ and $\Phi_{n}^{\text {store }}$, respectively). Then, $\phi_{s}\left(V_{B}(\mathcal{S})\right), \forall s \in \mathcal{S}$ is subjected to the following constraints:

- The sum of allocated payoffs should equal $V_{B}(\mathcal{S})$ :

$$
\sum_{s \in \mathcal{S}} \phi_{s}\left(V_{B}(\mathcal{S})\right)=V_{B}(\mathcal{S}) .
$$

- The payoff of a player $s$ in a coalition $\mathcal{S}$ should be at least equal to the payoff of its stand-alone action:

$$
\phi_{s}(\{s\}) \leq \phi_{s}\left(V_{B}(\mathcal{S})\right) .
$$

- A player $s$ cannot receive a higher payoff than its claim, so that fairness is preserved.

$$
0 \leq \phi_{s}\left(V_{B}(\mathcal{S})\right) \leq d_{s}
$$

\section{Bankruptcy game with Shapley Value (BS)}

In order to calculate the payoffs $\phi_{s}\{S\}$ of the described in Section III-B bankruptcy game, we use Shapley Value (SV) [20]. SV rewards a player $s$ with the SV payoff $\phi_{s}\left(V_{B}\right)$ according to its marginal contribution to the value of coalition, based on the characteristic function of eq. (15). In the present case, SV payoffs represent the contribution in withdrawing harvested or stored RE, depending on the considered $E$, when an $\mathcal{S}$ is formed. SV is based on four basic axioms [20]:

- Efficiency axiom: $\sum_{s \in \mathcal{S}} \phi_{s}\left(V_{B}\right)=V_{B}(\mathcal{S})$.

- Dummy axiom: If a player $s$ is such that $V_{B}(\mathcal{S})=V_{B}(\mathcal{S} \cup\{s\})$, for $\forall \mathcal{S}$ not containing $s$, it is $\phi_{s}\left(V_{B}\right)=0$.

- Symmetry axiom: If two players $s_{1}$ and $s_{2}$ are such that $V_{B}\left(\mathcal{S} \cup\left\{s_{1}\right\}\right)=V_{B}\left(\mathcal{S} \cup\left\{s_{2}\right\}\right)$, for $\forall \mathcal{S}$ containing player $s_{1}$ and $s_{2}$, then $\phi_{s_{1}}\left(V_{B}\right)=\phi_{s_{2}}\left(V_{B}\right)$.

- Additivity axiom: If $u$ and $v$ are characteristic functions, then $\phi(u+v)=\phi(v+u)=\phi(u)+\phi(v)$.

$\mathrm{SV}$, which displays a player's worth the studied game, when the player joins the coalition $\mathcal{S}$, is computed based on the chosen utility function $V_{B}$ of eq. (15), as

$$
\phi_{s}\left(V_{B}\right)=\sum_{\mathcal{S} \subseteq \mathcal{N} \backslash\{s\}} \frac{|\mathcal{S}| !(|\mathcal{N}|-|\mathcal{S}|-1) !}{|\mathcal{N}| !}\left[V_{B}(S \cup\{s\})-V_{B}(\mathcal{S})\right] .
$$

Table I

System Parameter VAlues

\begin{tabular}{|c|c|}
\hline Parameter & Value \\
\hline \hline$G_{t r x}$ & 6 \\
\hline$P_{n}^{c o n}$ & $130 \mathrm{~W}$ \\
\hline$\Delta_{P}$ & 4.7 \\
\hline$P_{n}^{t r}$ & $20 \mathrm{~W}$ \\
\hline Pathloss & $5 \mathrm{~d}$ B \\
\hline Shadowing fading & $-174 \mathrm{dBm}$ \\
\hline Noise power level & $24 \mathrm{~h}$ \\
\hline$T_{R E}$ & $5.14 \mathrm{kWh} / \mathrm{m}^{2} /$ day \\
\hline Solar radiation, BCN, June &
\end{tabular}

\section{Performance Evaluation}

We implemented a system-level simulator in MATLAB to examine the performance of BS. Section IV-A describes the considered simulation scenario and the respective performance evaluation results are presented in Section IV-B.

\section{A. Simulation Scenario}

We study a macrocell-sized area $A$ in Barcelona, where $\mathcal{N}=3$ MNOs have located and share their infrastructure, as described in Section II. A bandwidth of $20 M H z$ is used. Thus, $W_{n}=100 R B s$, while we set $S I N R_{\text {thresh }}=$ $-10 d B$ [14]. We use different MNO traffic profiles with $a_{n}=\{1.3,1.0,0.3\}$ for $n=1,2,3$, respectively, unless otherwise stated. A UE $k$ can have a data throughput demand $r_{k}=\{1024,512,256\} k b p s$, with random probability. We consider a standard solar generation system composed of $L=6 \mathrm{PV}$ panels, as described in [16], with system losses $\eta_{P V}=14 \%$ and inverter losses $\eta_{I N V}=10 \%$. A storage device composed of $M=3$ lithium batteries of $V=48 \mathrm{~V}$, $I=150 A h, D O D=80 \%$ and $\eta_{b}=15 \%$ is considered. The system is studied on the best day of the year in terms of solar insolation, i.e., June 21st. We focus on non-solar hours, considered as 20:00-07:00, during which stored RE (collected between 07:00-20:00 of the same day) has to be shared among cooperative MNOs of coalition $\mathcal{S}=\mathcal{N}$. Initial storage charge is set equal to 0 . The rest of our simulation parameters can be observed in Table I [13], [14], [16].

The proposed BS is compared with (i) Equal allocation (EQ), when each MNO $s \in \mathcal{S} \subseteq \mathcal{N}$ gets an equal share of the stored energy and (ii) Prioritised-claim allocation (PC), when MNOs are allocated with their complete claim of stored energy in a descending order.

Finally, we evaluate the performance of BS based on the hours of zero-grid-energy operation of the system $(h)$ and an indicator $\lambda_{n}$ of bought grid energy $x_{n}^{\text {grid }}(W h)$ in relation to each MNO's claim, defined as

$$
\lambda_{n}=\frac{x_{n}^{\text {grid }}}{d_{n}} .
$$

We assess the fairness of studied methods using the indicator of Jain's fairness index, calculated as

$$
J=\frac{\left(\sum_{n \in \mathcal{N}} \lambda_{n}\right)^{2}}{|\mathcal{N}| \sum_{n \in \mathcal{N}} \lambda_{n}^{2}} .
$$

\section{B. Performance Results}

Fig. 2 depicts the payoffs $\phi_{n}\left(V_{B}\right)$ of each MNO $n$ from the available stored energy for the different allocation strategies, as well as the energy transactions between each $n$ and the grid. 


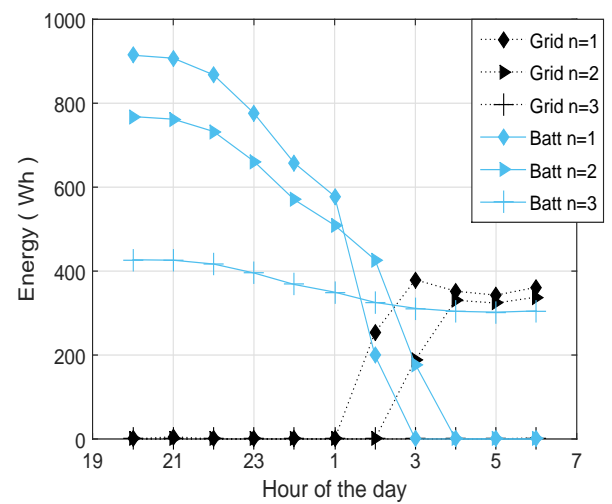

(a) Equal allocation

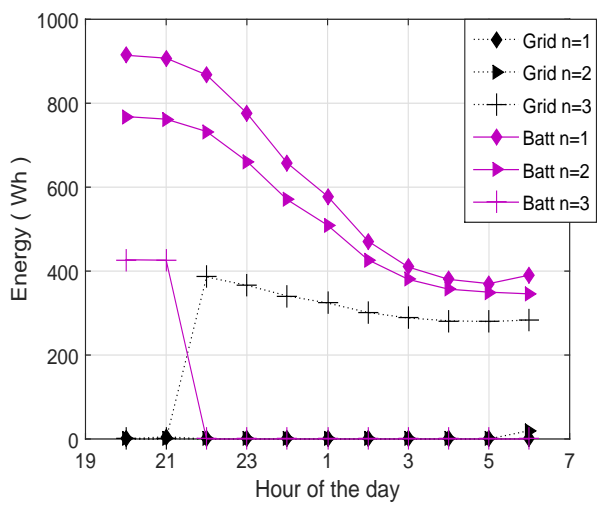

(b) Prioritised-claim allocation

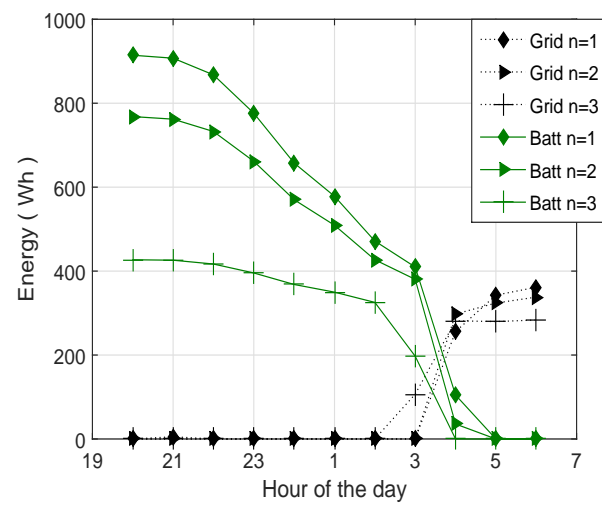

(c) Bankruptcy game with Shapley Value

Figure 2. Monitoring of energy throughout a day, Barcelona, 21st of June.

In detail, Fig. 2(a), Fig. 2(b) and Fig. 2(c) depicts the EQ, $\mathrm{PC}$ and proposed BS allocation of stored energy, respectively. According to Fig. 2(a), $n=1$ and $n=2$ are covered with their allocated stored energy for 5 and $6 h$, respectively. On the other hand, $n=3$ is grid-independent for all night hours and even ends up with a part of it unused at the end of the studied period. This is due to the fact that it gets the same amount of energy as $n=1,2$, even though its traffic volume is considerably lower. In Fig. 2(b), $n=1,2$ procure energy from the battery all night long, since they have the biggest claim and thus are awarded with the total amount of their energy needs. MNO $n=3$, though, is at disadvantage, since not a lot of stored energy had remained to provided it with grid-independence for more than $2 h$. Unlike EQ and PC, BS allocation offers a descent number of grid-independence hours to all cooperating MNOs. According to Fig. 2(c), when the bankruptcy game is applied, between 20:00 and 07:00, BS reassures 7, 7 and $6 h$ of grid-independence to $n=1,2,3$, respectively. After that, all MNOs use the remains of their allocated RE payoffs for one hour and complete their energy deficits by buying energy from the grid. BS manages to offer a respectable period of green network operation to all MNOs, since it considers both their cumulative energy needs and their marginal contribution to completely allocate the stored energy.

The exact amounts of stored and grid energy each $n \in \mathcal{N}$ receives when they have similar traffic patterns are apparent in Fig. 2. As can be seen in Fig. 3 though, we aim to capture the effectiveness of each method on reducing grid-energy consumption with indicator of eq. (20), $\lambda_{n}$, when $n=1$ has opposite traffic-peaks in comparison to $n=2,3$. According to the figure, EQ allocation results in larger percentage of bought grid-energy when the MNO's traffic is larger $(n=1)$, since it ignores any inequalities of traffic volume or energy needs of the MNOs. On the other hand, PC allocation minimizes griddependency for $n=1$ due to the fact that it has the largest traffic volume and thus the largest claim that gets fulfilled first. For the same reasons, $n=3$ buys almost $40 \%$ of its claims from the grid, even though it has the least traffic volume. Its percentage is considerably higher that the respective ones of $n=1$ and $n=2$, raising fairness issues of the method. Finally, BS captures the relation between each MNO's traffic and claims, leading them to buy similar amounts of grid-

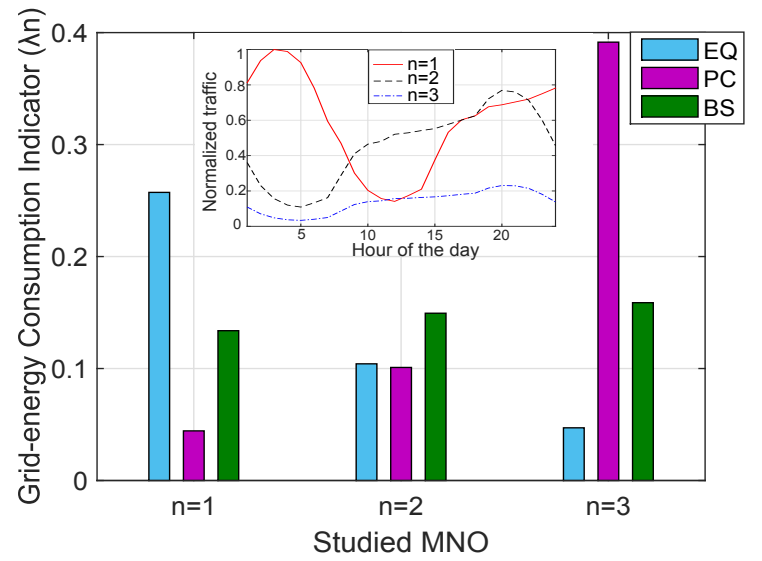

Figure 3. Indicator $\lambda_{n}$ of bought grid energy in relation to claimed energy.

energy. The absolute differences among bought grid-energy of MNOs are less than 0.025 .

Given that we considered in the previous figures MNOs with a specific traffic volume difference, which can affect performance of allocation methods, in Fig. 4 we see the effects of different traffic volumes on the fairness issues of each allocation method. More specifically, we vary traffic load factor $a_{3}$, while $a_{1}$ and $a_{2}$ remain unchanged, considering both similar and different traffic load peaks of $n=1$ and $n=2,3$ in Fig. 4(a) and Fig. 4(b), respectively. It is noticeable in both figures that Jain's fairness index of BS remains close to 1 irrespective of any traffic load volume or peak differentiations, since it considers each player contribution to sharing the stored energy. Compared to its counterparts, we notice that EQ allocation performs close to BS when traffic load peaks are similar (biggest gap equal to 0.09 when $a_{3}=0.2$ ), or even equally to BS in Fig. 4(a), when traffic load volume differences are alleviated, i.e., Jain's fairness index equal to 1 when $a_{3}>0.8$. The fact that MNOs have similar peaks does not allow traffic volume differences to affect to a big degree fairness of EQ allocation. However, when peaks are different in Fig. 4(b), fairness of EQ deteriorates and is outperformed by BS by a difference of 0.3 to 0.05 when $a_{3}=0.4$ and 1.6, respectively, since different traffic load peaks intensify differences in traffic volumes. Finally, PC allocation is far below EQ and BS in both cases, as there is always an MNO 


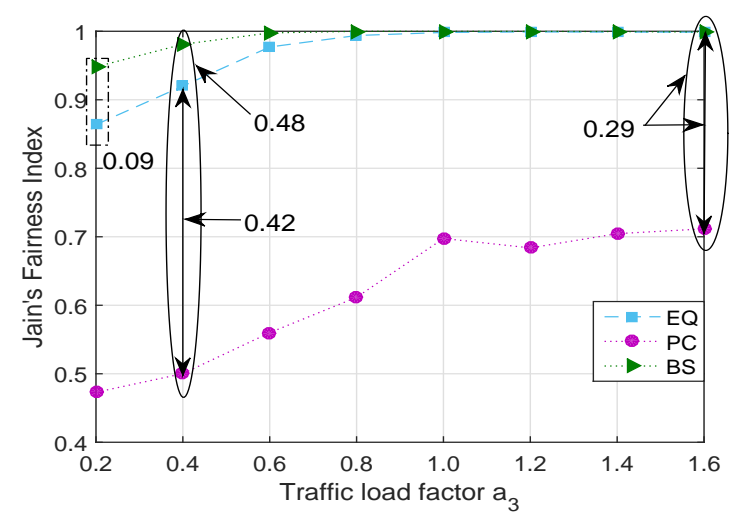

(a) Same traffic peaks for $n=1$ and $n=2,3$.

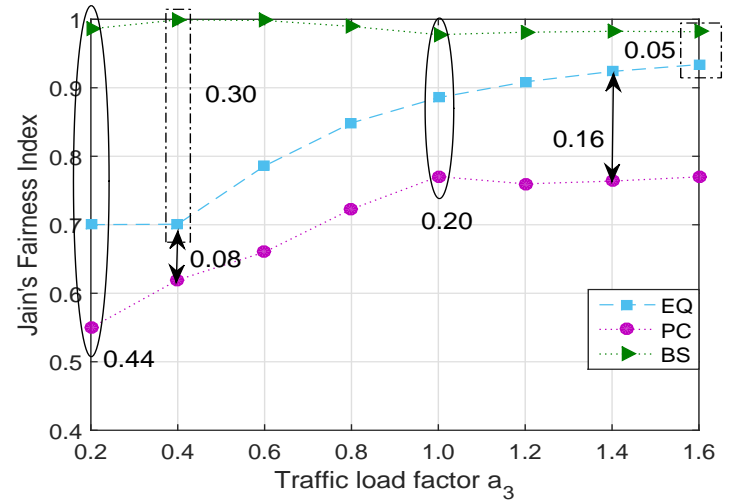

(b) Different traffic peaks for $n=1$ and $n=2,3$.

Figure 4. Jain's fairness index of studied allocation methods for different traffic load factor of MNO $n=3$.

in great need of grid energy. In more detail, difference gap of PC and BS is 0.48 and 0.29 when $a_{3}=0.4$ and 1.6 in Fig. 4(a) and 0.44 and 0.20 when $a_{3}=0.2$ and 1.0 , respectively, in Fig. 4(b). The respective gaps with EQ are 0.42 to 0.29 when $a_{3}=0.4$ and 1.6 in Fig. 4(a) and 0.08 and 0.16 when $a_{3}=0.4$ and 1.4, respectively, in Fig. 4(b). PC performs better when traffic peaks are different. The latter is attributed to the fact that more energy remains for the peak-time of $n=3$, since the other MNOs are in their low traffic period. In addition, when other MNOs are in their peak and reserve first their energy, $n=3$ is in its low traffic time and thus in less need of energy.

\section{CONCLUSIONS}

In this work, we studied sharing possibilities of stored solar energy among MNOs that share both their networks and RE infrastructure. Moreover, we focused on related fairness issues to further motivate cooperation among MNOs. We proposed an energy sharing method based on bankruptcy theory, which displayed that all cooperative MNOs could be provided with $6-7 h$ of green network operation with stored energy during night hours, regardless of their traffic. Furthermore, MNOs buy grid energy at similar percentages with our proposal, which was proved fairer in comparison to baseline methods by difference gaps of up to 0.30 and 0.48 for different MNO traffic profiles. As future work, we plan to assess the introduction of different RE sources to our RE infrastructure and energy transfer issues among MNOs with different RE and storage infrastructure.

\section{ACKNOWLEDGMENT}

The authors would like to thank the Research Projects AGAUR 2013-DI053, AGAUR (2014 SGR 1551), CellFive (TEC2014-60130-P) and COMANDER (612257).

\section{REFERENCES}

[1] "Cisco visual networking index: Global mobile data traffic forecast update, 2015-2020," Cisco, Feb. 2016.

[2] E. Oh, B. Krishnamachari, X. Liu, and Z. Niu, "Toward dynamic energyefficient operation of cellular network infrastructure," IEEE Commun. Mag., vol. 49, no. 6, pp. 56-61, Jun. 2011.

[3] M. Oikonomakou, A. Antonopoulos, L. Alonso, and C. Verikoukis, "Cooperative base station switching off in multi-operator shared heterogeneous network," in 2015 IEEE Global Communications Conference (GLOBECOM), Dec 2015, pp. 1-6.
[4] A. Antonopoulos, E. Kartsakli, A. Bousia, L. Alonso, and C. Verikoukis, "Energy-efficient infrastructure sharing in multi-operator mobile networks," IEEE Communications Magazine, vol. 53, no. 5, pp. 242249, May 2015.

[5] V. Chamola and B. Sikdar, "Solar powered cellular base stations: current scenario, issues and proposed solutions," IEEE Communications Magazine, vol. 54, no. 5, pp. 108-114, May 2016.

[6] M. Sheng, D. Zhai, X. Wang, Y. Li, Y. Shi, and J. Li, "Intelligent energy and traffic coordination for green cellular networks with hybrid energy supplies," IEEE Transactions on Vehicular Technology, vol. PP, no. 99, pp. 1-1, 2016.

[7] Y. K. Chia, S. Sun, and R. Zhang, "Energy cooperation in cellular networks with renewable powered base stations," IEEE Transactions on Wireless Communications, vol. 13, no. 12, pp. 6996-7010, Dec. 2014.

[8] J. Leithon, T. J. Lim, and S. Sun, "Energy exchange among base stations in a cellular network through the smart grid," in 2014 IEEE International Conference on Communications (ICC), Jun. 2014, pp. 4036-4041.

[9] J. Xu and R. Zhang, "CoMP meets smart grid: A new communication and energy cooperation paradigm," IEEE Transactions on Vehicular Technology, vol. 64, no. 6, pp. 2476-2488, Jun. 2015.

[10] J. Xu, L. Duan, and R. Zhang, "Cost-aware green cellular networks with energy and communication cooperation," IEEE Communications Magazine, vol. 53, no. 5, pp. 257-263, May 2015.

[11] D. Zordan, M. Miozzo, P. Dini, and M. Rossi, "When telecommunications networks meet energy grids: cellular networks with energy harvesting and trading capabilities," IEEE Communications Magazine, vol. 53, no. 6, pp. 117-123, Jun. 2015.

[12] J. Xu, L. Duan, and R. Zhang, "Energy group buying with loading sharing for green cellular networks," IEEE Journal on Selected Areas in Communications, vol. 34, no. 4, pp. 786-799, Apr. 2016.

[13] G. Auer, V. Giannini, C. Desset, and I. Godor, "How much energy is needed to run a wireless network?" IEEE Commun. Mag., vol. 18, no. 5, pp. 40-49, Oct. 2011.

[14] 3rd Generation Partnership Project, "Evolved universal terrestrial radio access (E-UTRA); radio frequency (RF) system scenarios," 3GPP TR 36.942, vol. 13.0.0, Rel. 13, Jan. 2016.

[15] A. Mesodiakaki, F. Adelantado, L. Alonso, M. D. Renzo, and C. Verikoukis, "Energy and spectrum efficient user association in millimeter wave backhaul small cell networks," IEEE Transactions on Vehicular Technology, vol. PP, no. 99, pp. 1-1, 2016.

[16] National Renewable Energy Laboratory. [Online]. Available: http://pvwatts.nrel.gov/index.php

[17] A. Jhunjhunwala, B. Ramamurthi, S. Narayanamurthy, J. Rangarajan, and S. Raj, "Powering cellular base stations; A quantitive analysis of energy options," Telecom centre of excellence (RiTCOE), Indian Institue of Technology, Madras, 2012.

[18] M. Pulido, J. Sánchez-Soriano, and N. Llorca, "Game theory techniques for university management: An extended bankruptcy model," Annals of Operations Research, vol. 109, no. 1, pp. 129-142, 2002.

[19] J. Alcalde, M. d. C. Marco, and J. A. Silva, "Bankruptcy games and the Ibn Ezra's proposal," Economic Theory, vol. 26, no. 1, pp. 103-114, 2005. [Online]. Available: http://dx.doi.org/10.1007/s00199-004-0527-z

[20] Z. Han, D. Niyato, W. Saad, T. Başar, and A. Hjorungnes, Game theory in wireless and communication networks: theory, models, and applications. Cambridge University Press, 2012. 